\title{
Satellite Link Layer Performance Using Two Copy SR-ARQ and Its Impact on TCP Traffic
}

\author{
Jing Zhu and Sumit Roy \\ Department of Electrical Engineering, University of Washington \\ Box 352500, Seattle, WA 98195, USA \\ \{zhuj, roy\}@ee.washington.edu
}

\begin{abstract}
The paper focuses on improving performance of land mobile satellite channels (LMSC) at higher frequencies such as K or EHF band, where shadowing is the primary impediment to reliable data transmission. Compared with short-term multipath fading, shadowing is characterized by longer time constants so that interleaving is not desirable as it introduces unacceptably large delays. To combat error bursts, an adaptive two-copy SR-ARQ scheme is proposed that uses a suitable $d e$ lay between every retransmission. Closed-form solutions for metric of interest:mean transmission time, success probability, and residual loss probabilityare derived and validated by simulation. An optimal choice of the delay is determined and the performance of TCP traffic over such a link layer is evalauted by simulation and compared to normal SR-ARQ in terms end-to-end throughput.
\end{abstract}

\section{Introduction}

Currently, there is a great deal of interest in extending satellite communications to higher bands ( $\mathrm{K}$ or EHF band) in order to achieve more transmission bandwidth. In [3] it was shown that the primary impediment to the land mobile satellite channel at $\mathrm{K}$ or EHF bands is shadowing due to blockage rather than multipath fading. In such cases, the channel can be represented by a two-state Markov process as in [1]. In bad (shadowed) states, the average SNR is too low to correctly transmit signals even with powerful forward error correction (FEC) codes while in good (unshadowed) states, the large value of Rice factor corresponding to the line-of-sight component guarantees reliable signal transmission even without much FEC protection. As is well known, interleaving is widely used with FEC to resist fading and improve the reliability of a wireless channel with burst errors. However, with increasing average length of error bursts, the interleaving depth needed may lead to unacceptable end-to-end delays. Therefore, a multiple copy (re)transmission scheme was proposed in [2], that inserts a suitable delay between copies of the transmitted packet. This paper extends the idea to a satellite channel with shadowing by modifying how the number of copies is varied with retransmission number in the interest of stability. For the two-copy 
case, simple expressions for the metrics of interest (i.e. mean transmission time, transmission success probability, and residual loss probability) are obtained.

Such adaptive transmission schemes [4] have naturally been considered since most wireless satellite channels such as LMSC are time-varying. In such methods, the coding rate, packet length and retransmission mode parameters etc. can be varied to match the transmitter for current channel conditions. Nevertheless, their performance depends critically on the efficiency and accuracy of channel state estimation (CSE) at the receiver that is fed back to the transmitter. Obviously,the long propagation delay of a satellite link implies that all variations less than one round trip time cannot be tracked. However, when average shadowing periods typically exceed a round-trip time, a long-term estimate of the average length of shadowing periods may be used to determine the optimal delay of our proposal.

The paper is organized as follows. In the next section, expressions for success probability for each transmission, mean transmission time, and residual loss probability are derived for delayed two-copy (DTC) SR-ARQ. Section 3 contains numerical results to quantify the improvements of the proposal. The impact of this improved LL design on end-to-end TCP throughput is assessed by comparing with normal SR-ARQ as well under the assumption of the same allowable copy number. Finally, we conclude the paper in Section 4.

\section{Delayed Two-Copy (DTC) SR-ARQ}

The protocol employs the basic selective repeat (SR-ARQ) strategy, except that two identical copies of a packet with a delay $D$ is sent at each attempt (note that an attempt consists of transmissions or retransmissions of the packets). Only when both copies in an attempt are lost, a negative acknowledgment is produced and retransmission occurs. Compared with a normal 1-copy scheme, the equivalent code rate is thus 0.5 .

The parameters used in the subsequent analysis are as follows:

$X$ : Good-state time share parameter;

Peg: Packet error rate in good states;

Peb: Packet error rate in bad states;

$m$ : The Mean length of bad states;

RTT: Round trip time of satellite channel;

$B w$ : Bandwidth of a satellite channel.

The usual alternating two-state Markov model is assumed to represent the channel state evolution in time; the duration of a 'bad' or shadowed state (i.e. the error burst length) is exponentially distributed with mean $m$. In the 'bad' or shadowed state, it is reasonable to assume $P e b \approx 1$, implying that no successful transmission is possible during shadowing. Further, $P e g<<P e b$ and for analytical purposes, may be assumed equal to zero, i.e. all packet transmissions during 
'good' or un-shadowed state are successfully received. This leads to the important simplification that the sequence of packet success and failures is a Markov process $^{1}$.

First we consider transmission of any two successive packets with a general delay $(d)$ in between. We define an attempt to imply transmission of two copies (of the same packet) as per our scheme. We now identify two important situations: (i) the success/failure of the second copy in an attempt is related to that of the first copy in the same attempt; (ii) the success/failure of the first copy in current attempt is related to success/failure of the second copy in the previous attempt. Hence there are two relevant values of $d$ for consideration - D and RTT.

Since the packet error rate is negligible in good states, packet losses take place in only bad states. If the previous copy is lost, the probability of correctly receiving the current one can be written as:

$$
P\{S \mid F\}=P\left\{B_{E}, G\right\} P\left\{S_{G}\right\}=P\left\{B_{E}\right\} P\left\{G \mid B_{E}\right\} P\left\{S_{G}\right\},
$$

where the notations represent the following events:

$S$ : Success on the current copy;

$F$ : Failure on the previous copy;

$B_{E}$ : The bad state during transmission of the previous copy is completed before transmission of the current copy;

$G$ : The current copy is transmitted in a good state;

$S_{G}$ : Success of the current copy in a good state.

Since the duration of bad state is exponentially distributed with the mean of $m$ and the inter-duration between two copies is $d$, we have

$$
P\left\{B_{E}\right\}=1-e^{-\frac{d}{m}} .
$$

and clearly,

$$
P\left\{S_{G}\right\}=1-P e g .
$$

An exact expression for $P\left\{G \mid B_{E}\right\}$ is difficult and therefore an intuitive approximation is given next. We know that $P\left\{G \mid B_{E}\right\}$ is a function of $d$. Let's consider two extreme cases:

For $d \rightarrow$ 0, i.e., two copies are sent next to each other with no delay. Since the end of the bad state for the previous transmission is followed by a good state, the current copy will be sent in the good state with probability 1 , leading to $P\left\{G \mid B_{E}\right\} \rightarrow 1$. (Here we ignore the event that the good state ends before one LL packet duration because the good state mean duration is significantly longer than a LL packet duration).

For $d \rightarrow \infty$, the correlation between the channel states for the two copies vanishes, leading to $P\left\{G \mid B_{E}\right\} \rightarrow P\{G\}$, where $P\{G\}=X$.

\footnotetext{
${ }^{1}$ In general, the sequence of packet success and failures is a Hidden Markov process, and not strictly a Markov process.
} 
We define the above two extreme cases as two mutually exclusive events: the channel state for the current copy is completely correlated $(C C)$ or completely un-correlated $(C U)$ to that for the previous one. The probability of $C C$ is given by the correlation function of $e^{-\frac{d}{m}}\left(=\left\{\begin{array}{l}1, d=0 \\ 0, d=\infty\end{array}\right)\right.$, and the probability of $C U$ is $1-e^{-\frac{d}{m}}$. The desired result for $P\left\{G \mid B_{E}\right\}$ is given by the statistical average of the above, i.e.,

$$
P\left\{G \mid B_{E}\right\} \approx 1 \cdot e^{-\frac{d}{m}}+X \cdot\left(1-e^{-\frac{d}{m}}\right) .
$$

In conclusion, the probability of correctly receiving the current copy given that the previous is lost is expressed as

$$
P_{S e}(d)=\left(1-e^{-\frac{d}{m}}\right)\left(e^{-\frac{d}{m}}+X\left(1-e^{-\frac{d}{m}}\right)\right)(1-P e g), d=\{D, R T T\} .
$$

We next consider the success probability for transmission and retransmission, denoted by $P_{S t}$ and $P_{S r}$ respectively. There are following events involved:

$S_{1}$ : Success on the first copy;

$F_{1}$ : Failure on the first copy;

$S_{2}$ : Success on the second copy;

$F_{2}$ : Failure on the second copy;

$F_{o}$ : Failure on all previous attempts.

Obviously,

$$
P\left\{S_{2} \mid F_{1}\right\}=P_{S e}(D),
$$

and the state for the current copy only depends on that for the previous copy, leading to

$$
P\left\{S_{1} \mid F_{o}\right\}=P_{S e}(R T T)
$$

The first copy of the transmission has probability of $X(1-P e g)$ of being correctly received. Therefore,

$$
\begin{aligned}
P_{S t} & =P\left\{S_{1}\right\}+P\left\{F_{1}\right\} P\left\{S_{2} \mid F_{1}\right\} \\
& =X(1-P e g)+(1-X(1-P e g)) P_{S e}(D) .
\end{aligned}
$$

Then

$$
\begin{aligned}
P_{S r} & =P\left\{S_{1} \mid F_{o}\right\}+P\left\{F_{1} \mid F_{o}\right\} P\left\{S_{2} \mid F_{1}, F_{o}\right\} \\
& =P\left\{S_{1} \mid F_{o}\right\}+P\left\{F_{1} \mid F_{o}\right\} P\left\{S_{2} \mid F_{1}\right\} \\
& =P_{S e}(R T T)+\left(1-P_{S e}(R T T)\right) P_{S e}(D) .
\end{aligned}
$$

If the maximum number of attempts allowed is $N$, the packet loss probability $P_{l}^{(N)}$ after $N$ attempts is

$$
P_{l}^{(N)}=\left(1-P_{S r}\right)^{N-1}\left(1-P_{S t}\right)
$$


Denote by $T$ the time from the first transmission to receipt of the acknowledgement. We have the following results of probability distribution function of $T$ :

1)Correctly receiving the second copy:

$$
\mathbf{P}[T=i(R T T+D)]= \begin{cases}(1-X(1-P e g)) P_{S e}(D) & i=1 \\ \left(1-P_{S t}\right)\left(1-P_{S r}\right)^{i-2}\left(1-P_{S e}(R T T)\right) P_{S e}(D) & i \geq 2\end{cases}
$$

2) Correctly receiving the first copy:

$$
\mathbf{P}[T=i R T T+(i-1) D]= \begin{cases}X(1-P e g) & i=1 \\ \left(1-P_{S t}\right)\left(1-P_{S r}\right)^{i-2} P_{S e}(R T T) & i \geq 2\end{cases}
$$

Thus, the mean value of $T$ is given below

$$
\begin{aligned}
E[T]= & \frac{1}{\left(1-P_{l}^{(N)}\right)} \sum_{i=1}^{N}\{(i R T T+(i-1) D) \mathbf{P}[T=i R T T+(i-1) D]+ \\
& i(R T T+D) \mathbf{P}[T=i(R T T+D)]\}
\end{aligned}
$$

As $N \rightarrow \infty, P_{l}^{(N)} \rightarrow 0$ and we get a closed-form solution for (13) as follows:

$$
\begin{aligned}
\lim _{N \rightarrow \infty} E[T]= & \frac{\left(1-P_{S t}\right)}{P_{S r}}\left[R T T+D+R T T P_{S r}+D\left(1-P_{S e}(R T T)\right) P_{S e}(D)\right] \\
& +X(1-P e g) R T T+(1-X(1-P e g)) P_{S e}(D)(R T T+D)(14)
\end{aligned}
$$

The analytical expressions of Eq.10 and Eq.14 are most useful as they provide performance estimate for applications with special QoS requirements. Of course, the mean error burst length must be obtained a-priori in practice using a suitable long-term channel estimator. In the following, we will assume that $m$ is known.

\section{$3 \quad$ Numerical Results}

In all simulations and analysis reported here, a link layer packet duration is chosen as the unit of time. Wireless channel bandwidth $(B w)$ is fixed at $1 \mathrm{Mbps}$.

\subsection{Performance Comparison of DTC-SR-ARQ to Interleaving}

In this section, we consider an error burst with fixed length $m$ (packets) followed by a sufficiently long error-free period. The metric of interest is additional delay, defined as the extra delay introduced by the method employed to resist fading (DTC-SR-ARQ or Interleaving).

For DTC-SR-ARQ, the minimum delay inserted between two copies of a packet for a successful transmission is $m-1$. The additional delay of the first copy is zero while that of the second one is $m$. Since either of them are equally likely to be transmitted during an error burst, the average additional delay introduced by DTC-SR-ARQ is $\frac{m}{2}$. 

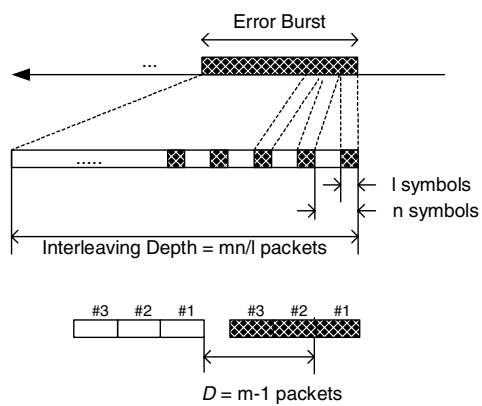

Fading Channel

a) Interleaving with FEC code $(n, k, l)$

Error $\square$ Correct

Fig. 1. Comparison of DTC-SR-ARQ to Interleaving in terms of Additional Delay

For interleaving with $\mathrm{RS}(\mathrm{n}, \mathrm{k}, \mathrm{l})$, the minimum interleaving length for error free transmission is $\frac{n}{l} m$ (packets), where $n$ is the length of codeword, $k$ is the number of information symbols in a codeword, and $l$ is the maximum number of correctable symbols in a codeword. De-interleaving starts at receiver only after receiving all $\frac{n}{l} m$ packets, thus the additional delay is $\frac{n}{l} m$ for the first packet, and zero for the last one. The consequent average additional delay introduced by interleaving is $\frac{n}{2 l} m$. Fig. 2 demonstrates that the additional delay as a function of $m$ is shorter for DTC-SR-ARQ than interleaving, with difference increasing for longer error bursts.

\subsection{Delay Optimization of DTC-SR-ARQ}

Fig.3 shows the success probability of the first transmission as a function of the delay $D$ - our analytical results match simulations quite well. From Fig.3, we

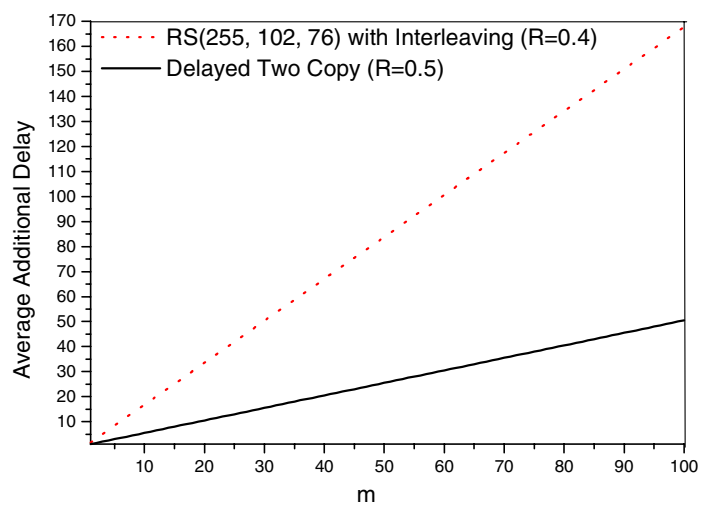

Fig. 2. Delay Comparison of Interleaving and DTC-SR-ARQ 


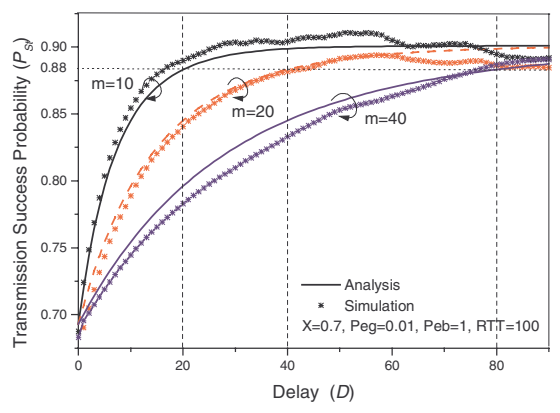

Fig. 3. Success Probability of Transmission

also see that longer error bursts need longer delay to achieve the same success probability.

Fixing the maximum number of attempts at 3, we study the residual loss probability after retransmission in Fig.4a); the residual loss probability is dramatically reduced by increasing delay. Fig.4b) investigates the mean transmission time $T$ and indicates that there exists an optimal value of delay yielding the minimum mean transmission time.

The optimal delay for achieving the minimum average transmission time, using the first-order necessary conditions, is given by $\frac{d T}{d D}=0$. However, it is tedious to explicitly solve. Figs.1-3 show that $D=2 m$ is a good pragmatic choice considering $P_{T s}, P_{l}^{(N)}$, and $T$. Therefore, in our following simulation on TCP performance, we will use $D=2 \mathrm{~m}$.

Fig.5 studies the link layer performance of DTC-SR-ARQ with $D=2 \mathrm{~m}$ in terms of residual packet loss probability and mean transmission time. Analytical results indicate that longer average burst error length leads to higher residual packet loss probability and longer mean transmission time. We also compare these results with normal SR-ARQ for the same maximum copy number (MCN)

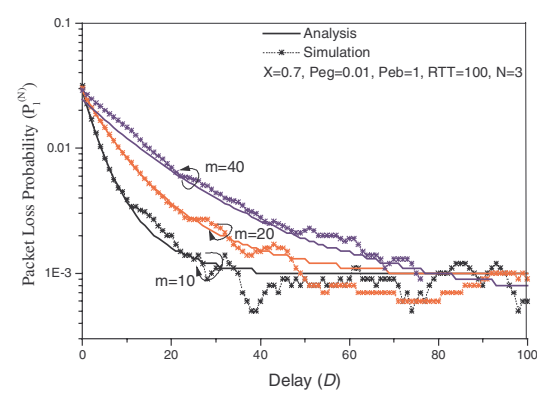

a)

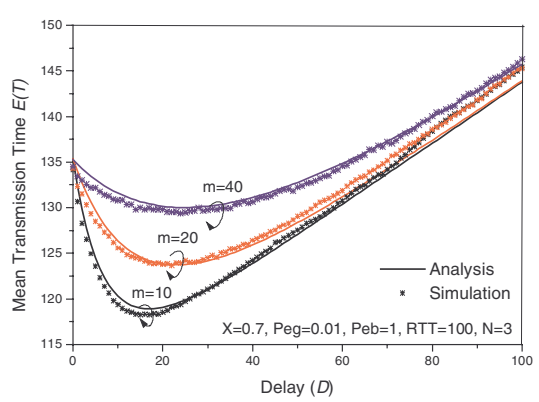

b)

Fig. 4. Packet Loss Probability a) and Mean Delay b) after $N$ attempts $(N=3)$ 


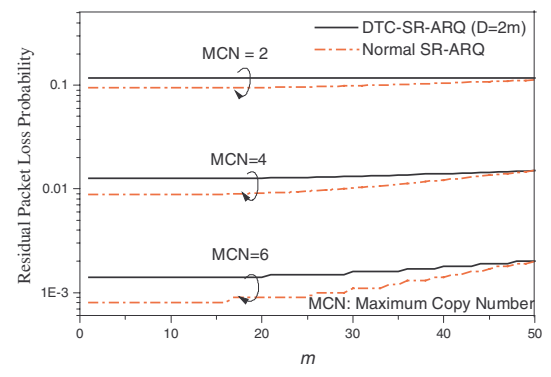

a) Residual Packet Loss Probability After $N$ Attempts

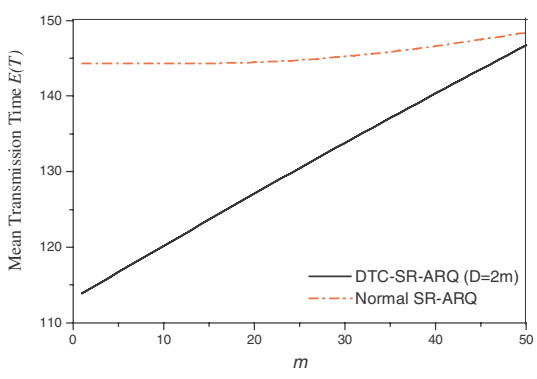

b) Mean Transmission Time $(N=\infty)$

Fig. 5. Performance for $D=2 m(R T T=100, X=0.7, P e g=0.01)$

so that the maximum transmission number is MCN (say 4) for SR-ARQ and MCN/2 (say 2) for DTC-SR-ARQ. It is seen that by using DTC-SR-ARQ the mean transmission time is significantly reduced at the expense of a small increase in residual packet loss probability. Furthermore, shorter the average length of error bursts, the more the improvement in mean transmission time. In addition, by using DTC-SR-ARQ instead of normal SR-ARQ, we can reduce maximum

transmission time from $M C N \times R T T$ to $M C N \times \frac{(R T T+D)}{2}$. If $m<<R T T$, we have $R T T>D$ because of $D=2 m$, leading to almost $50 \%$ reduction in maximum transmission time.

\subsection{On TCP Performance}

In this section, we study the performance of TCP over two-copy delayed SRARQ. The delay is bounded by half the RTT and set as $D=\min \left(2 m, \frac{R T T}{2}\right)$. Assuming a fixed maximum number of retransmission attempts(say 8), the maximum transmission time for normal is 7 and 3 RTTs respectively for regular SRARQ and our two-copy delayed SR-ARQ. Fig.6 shows that the TCP end-to-end throughput is improved by using our scheme, especially when the average error burst length is short. When the error burst length increases, the performance improvement using our proposal is reduced. In other words, the two-copy delayed SR-ARQ is more suitable for the fast shadow fading channel with much shorter error burst length compared to the round trip time.

\section{Conclusion}

In this paper, we proposed an optimized two-copy delayed SR-ARQ scheme for the shadowed satellite channel in the K or EHF bands. Shadowing leads to longer fade durations compared with multipath fading; consequently multiple copy transmission with a delay was suggested in place of interleaving to combat burst errors. Analytical results showed that success probability of each transmission is significantly improved, and mean transmission time is reduced as well. 


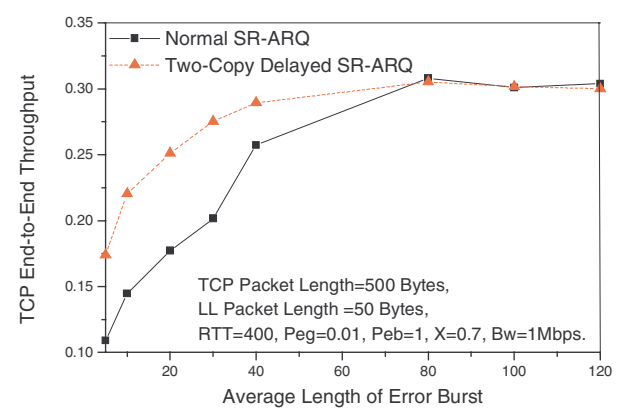

Fig. 6. TCP End-to-End Throughput Comparison (Buffer Size=20000 bytes)

Simulations performed to compare our scheme with normal SR-ARQ in terms of TCP end-to-end throughput indicate that our proposal achieves noticeable performance improvement especially for the fast shadowing channels with error burst longer than a link layer packet but shorter than one round trip time.

\section{References}

[1] E. Lutz, D. Cygen, M. Dippold, F. Dolainsky, and W. Papke, "The Land Mobile Satellite Communication Channel - Recording, Statistics, and Channel Model, IEEE Trans. on Vehicular Tech.” , Vol . 40, No. 2, May 1991, pp.375-386. 909

[2] J. Zhu, Z. Niu, Y. Wu, "A delayed multiple copy retransmission scheme for data communication in wireless networks" ,Proc. Ninth IEEE International Conference on Networks, 2001 pp. 310 -315. 909

[3] J. B. Schodorf, "EHF Satellite Communications on The Move: Baseband Considerations," MIT Lincoln Lab Technical Report 1055, Feb. 2000. 909

[4] A. Annamalai, V.K. Bhargava, "Analysis and Optimization of Adaptive Multicopy Transmission ARQ Protocols for Time-Varying Channels," IEEE Trans. on Commu., vol. 46, no. 10, pp. 1356-1368 910 